\title{
BMJ Open Blood pressure and cardiac output during caesarean delivery under spinal anaesthesia: a prospective cohort study
}

\author{
Trond Melbye Michelsen (ID ,1,2 Christian Tronstad, ${ }^{3}$ Leiv Arne Rosseland ${ }^{2,4}$
}

To cite: Michelsen TM, Tronstad C, Rosseland LA. Blood pressure and cardiac output during caesarean delivery under spinal anaesthesia: a prospective cohort study. BMJ Open 2021;11:e046102. doi:10.1136/ bmjopen-2020-046102

- Prepublication history for this paper is available online. To view these files, please visit the journal online (http://dx.doi. org/10.1136/bmjopen-2020046102).

Received 21 October 2020 Accepted 26 May 2021

Check for updates

(C) Author(s) (or their employer(s)) 2021. Re-use permitted under CC BY-NC. No commercial re-use. See rights and permissions. Published by BMJ.

${ }^{1}$ Department of Obstetrics, Oslo University Hospital, Oslo, Norway ${ }^{2}$ Institute of Clinical Medicine, Faculty of Medicine, University of Oslo, Oslo, Norway

${ }^{3}$ Department of Clinical and Biomedical Engineering, Division of Technology and Innovation, Oslo University Hospital, Oslo, Norway

${ }^{4}$ Department of Research and Development, Division of Emergencies and Critical Care, Oslo University Hospital, Oslo, Norway

Correspondence to Professor Trond Melbye Michelsen; trmi1@ous-hf.no

\section{ABSTRACT}

Objectives We have previously established a method to measure transfer of nutrients between mother, placenta and fetus in vivo. The method includes measurements of maternal and fetal blood flow by Doppler ultrasound prior to spinal anaesthesia. Spinal anaesthesia affects maternal blood pressure and cardiac output. We aimed to determine the effect of spinal anaesthesia in mothers undergoing an elective caesarean section on blood pressure, heart rate and cardiac output, and whether cardiac output levels were comparable before induction of spinal anaesthesia and before delivery.

Design Prospective cohort study.

Setting Tertiary hospital in Norway.

Participants 76 healthy women with uneventful pregnancies undergoing an elective caesarean section. Interventions We induced spinal anaesthesia with a standard prevention of hypotension including intravenous fluid coloading and phenylephrine infusion.

Primary and secondary outcome measures Primary outcome measure was maternal cardiac output, and secondary outcome measures were invasive systolic blood pressure and heart rate. We measured heart rate and blood pressure by continuous invasive monitoring with a cannula in the radial artery. Cardiac output was estimated based on continuous arterial waveform. We compared maternal parameters $30 \mathrm{~s}$ before induction of spinal anaesthesia to 30 s before delivery.

Results Median age at delivery was 34.5 (range 21-43) years and 17 of 76 women were nulliparous. The most prevalent indications were previous caesarean section and maternal request. Among 76 included women, 71 had sufficient data for analysis of endpoints. Median cardiac output was 6.51 (IQR (5.56-7.54) L/min before spinal anaesthesia and $6.40(5.83-7.56) \mathrm{L} / \mathrm{min}$ before delivery $(p=0.40))$. Median invasive systolic blood pressure increased from $128.5(120.1-142.7) \mathrm{mm} \mathrm{Hg}$ to 134.1 (124.0-146.6) mm Hg ( $p=0.014)$, and mean heart rate decreased from 86.0 (SD 13.9) to 75.2 (14.2) $(p<0.001)$. Conclusions Maternal cardiac output at the time of caesarean delivery is comparable to levels before induction of spinal anaesthesia.

Trial registration number NCT00977769.

\section{INTRODUCTION}

In healthy human pregnancies, the uteroplacental vessels are transformed into lowresistance channels through which maternal

\section{Strengths and limitations of this study}

- We performed continuous invasive monitoring of maternal vital functions.

- We followed a standardised phenylephrine protocol to counteract spinal anaesthesia-induced hypotension.

- Uteroplacental blood flow was not measured after induction of spinal anaesthesia.

blood can enter the intervillous space with low force. ${ }^{1}$ The uteroplacental circulation is through evolution established to maintain stable blood flow to support fetal growth and nutritional needs. ${ }^{2}$

We have previously established a method to measure transfer of nutrients between mother, placenta and fetus in vivo. ${ }^{34}$ The method includes measurements of maternal and fetal blood flow by Doppler ultrasound prior to spinal anaesthesia, and thereafter measurements of nutrients in maternal radial artery and uterine vein and umbilical artery and vein while the uteroplacental circulation is still running. The measurements of blood flow are based on the Doppler ultrasound measurements, and may therefore be affected by changes in maternal cardiac output $(\mathrm{CO})$ due to spinal anaesthesia.

Spinal anaesthesia is preferred for an elective and acute caesarean section due to a favourable safety profile compared with general anaesthesia. ${ }^{5}$ Spinal anaesthesia minimises risk of failed intubation, ventilation and aspirations. ${ }^{6}$ However, spinal anaesthesia affects maternal $\mathrm{CO}$ and blood pressure, and maternal side effects including hypotension, nausea and vomiting are well known. ${ }^{7}$ Substandard management of spinal anaesthesia-induced hypotension is still a cause of maternal morbidity and mortality, ${ }^{8}$ and prophylaxis and treatment with vasoconstrictive drugs are recommended. ${ }^{9}$ Furthermore, low maternal blood pressure and $\mathrm{CO}$ 
may affect uteroplacental perfusion and thereby fetal well-being.

Robson et $a l^{10}$ showed that maternal CO was affected to a greater degree in mothers who received spinal anaesthesia compared with epidural anaesthesia, and this difference was related to neonatal acid-base status. However, in their series of 32 caesarean sections, no fetus was depressed due to the anaesthesia regimen. ${ }^{10}$

Hypotension during spinal anaesthesia has been described as almost inevitable. ${ }^{11}$ However, several studies have addressed prevention of hypotension during spinal anaesthesia. We have previously documented that bupivacaine $(7 \mathrm{mg}$ compared with $10 \mathrm{mg}$ ) has dose-dependent hypotensive effects, which was opposed by a low-dose infusion of phenylephrine $(0.25 \mu \mathrm{g} / \mathrm{kg} / \mathrm{min})$ compared with placebo, in a randomised controlled trial using continuous invasive monitoring of mothers during caesarean section. ${ }^{12}$ Although hypotension may be counteracted by vasopressor administration, no study has previously evaluated the effect on invasively measured CO during caesarean section.

The primary aim was to determine changes in maternal $\mathrm{CO}$ at time of delivery compared to prior to spinal anaesthesia in healthy women undergoing an elective caesarean section. Secondary aims were to determine changes in maternal blood pressure and heart rate (HR). We hypothesised that the departmental procedure of spinal anaesthesia with low-dose bupivacaine combined with a low-dose phenylephrine bolus+infusion, keeps CO, blood pressure and HR stable, and that measurements of maternal vital parameters before induction of spinal anaesthesia are comparable to the measurements before delivery.

\section{MATERIALS AND METHODS}

The patients participated in a randomised, doubleblinded, placebo-controlled, parallel-group comparison of carbetocin and oxytocin given intravenously during an elective caesarean delivery under spinal anaesthesia. The study was conducted at the Department of Anaesthesiology, Oslo University Hospital, Rikshospitalet between November 2009 and September 2011. The results from the randomised controlled trial have been published previously. ${ }^{13}$ The project is completed, the dataset is anonymised and available open access (uploaded in Mendeley). This protocol is a substudy planned and performed after closure of the primary project and performed on an open access dataset. The data included in this analysis were not part of the above-mentioned RCT and has not been published previously.

Eligible participants were screened by the senior author (LAR) for inclusion at their last midwife consultation before their scheduled delivery. Written informed consent was obtained before inclusion. The participants were specifically informed of the extra pain caused by the arterial cannulation and that this was due to research purposes only. The inclusion criteria included singleton pregnancy, being aged 18 years or older and a scheduled planned caesarean section at $\geq 36$ weeks of gestation. The exclusion criteria included pre-eclampsia, placenta praevia, placenta accreta, von Willebrand disease or other bleeding disorder, known intolerance to oxytocin or carbetocin and systolic arterial pressure (SAP) $<90 \mathrm{~mm}$ Hg. A total of 185 patients were screened, and 76 were included in the study (figure 1). The indications for an elective caesarean section were maternal request $43 \%$, previous caesarean delivery $25 \%$, breech presentation $8 \%$, other obstetric reasons $13 \%$ and other non-obstetric maternal or neonatal medical conditions $11 \%$.

In addition to continuous invasive beat-to-beat haemodynamic variables that were collected and stored electronically, we registered each patient's age, height, weight, hours in fasting for solids, estimated intake of liquids and gestational age. Maternal weight on inclusion was used to calculate the body mass index (BMI; weight in $\mathrm{kg}$ / (height in metres) $)^{2}$ ). An arterial catheter was placed into the radial artery after infiltration of lidocaine $(10-20 \mathrm{mg})$ immediately after arrival at the operating theatre. Invasive blood pressure was calibrated and continuous beat-to-beat measurements of invasive SAP and HR were recorded. CO was recorded using PulseCO (PulseCO, Cambridge, UK), an integrated part of the LiDCO plus monitoring system (LiDCO, Cambridge, UK). Baseline values of SAP, mean arterial pressure (MAP), diastolic arterial pressure (DAP) and HR represent a $60 \mathrm{~s}$ mean of measurements with the patient in left lateral position. We omitted the calibration of $\mathrm{CO}$ with the lithium dilution technique since the primary outcome was changes in repeated measurements of haemodynamic variables. Other haemodynamic variables were not included in this analysis but are available on request. Hence, the primary outcome was change of maternal CO from before induction of spinal anaesthesia compared with prior to delivery. Secondary outcomes were changes in maternal blood pressure and HR.

Spinal anaesthesia was induced with the L2-L3 vertebral interspace as the targeted location. The woman was placed in a right lateral position. Isobaric bupivacaine $10 \mathrm{mg}$ (Marcain Spinal, Aspen Pharma, Ireland)+fentanyl $20 \mu \mathrm{g}$ was injected through a $25 \mathrm{G}$ non-traumatic needle (Pencan, B. Braun, Melsungen, Germany). Coloading with a rapid intravenous infusion of $0.9 \%$ saline $\left(37^{\circ} \mathrm{C}\right.$, $10 \mathrm{~mL} / \mathrm{kg}$, duration $10-15 \mathrm{~min}$ ) and a phenylephrine bolus $(0.25 \mu \mathrm{g} / \mathrm{kg})$, followed by a phenylephrine infusion $(0.25 \mu \mathrm{g} / \mathrm{kg} / \mathrm{min})$, were given. During caesarean section, the patient was kept in supine position with an operating wedge under her right hip (19 ${ }^{\circ}$ Tempur pillow). Hypotension ( $\mathrm{SAP}<90 \mathrm{~mm} \mathrm{Hg}$ ) was treated with an extra intravenous bolus of phenylephrine $30 \mu \mathrm{g}$ if the $\mathrm{HR}$ was $>60$ beats/min or with intravenous ephedrine $5-10 \mathrm{mg}$ if the HR was $\leq 60$ beats/min.

\section{Patient involvement}

The study was planned in 2009, and the protocol did not include patient involvement. 
Figure 1

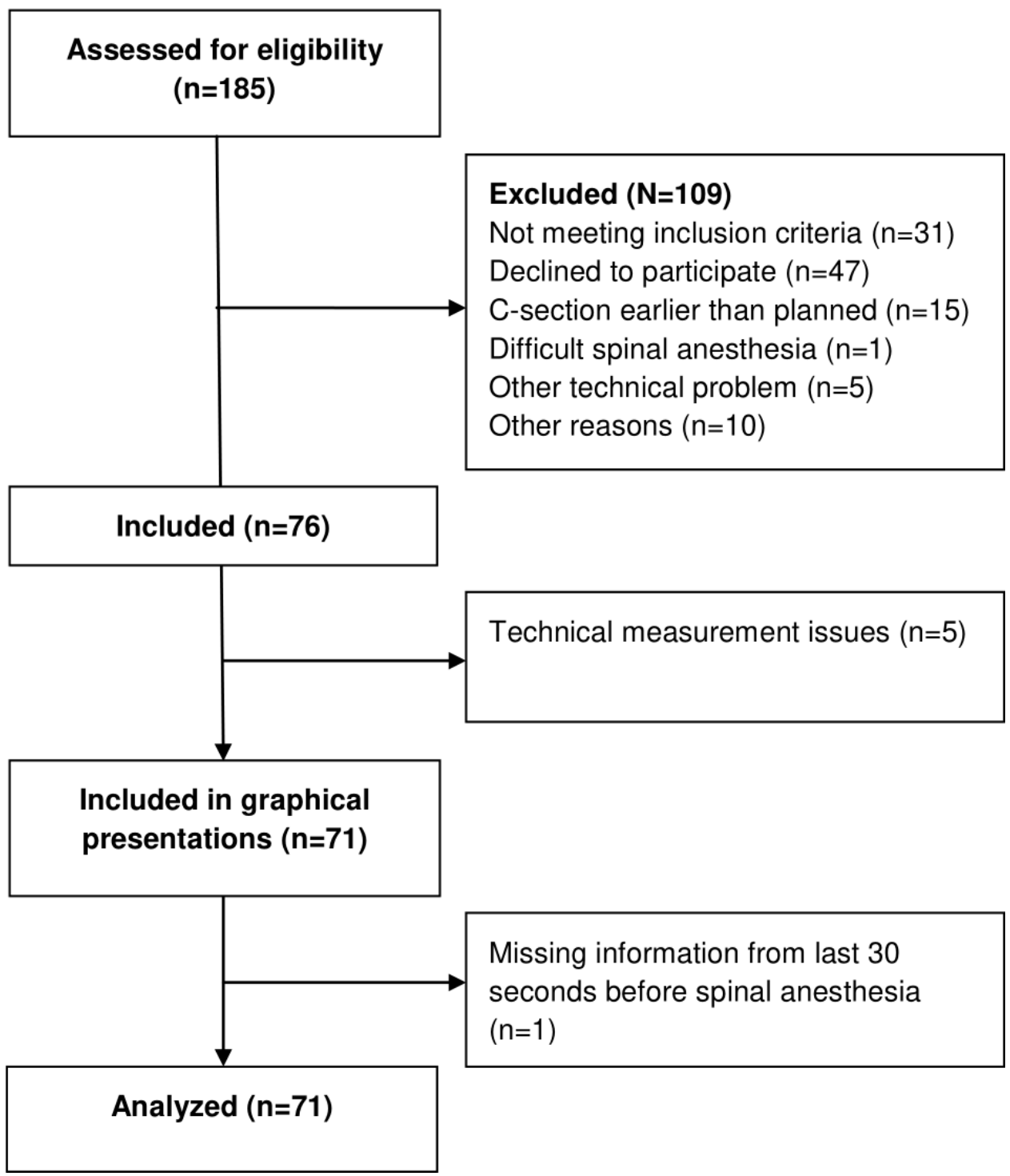

Figure 1 Flowchart showing inclusion of participants. In the original study, 185 participants were approached for inclusion, and 76 participated (13). In this study, 5 more participants were excluded due to technical measurement issues, thus leaving 71 participants for graphical presentations and 70 for statistical analysis.

\section{Statistical analyses}

The raw data contained one sample of SAP, HR and CO per beat, with different recording lengths for each participant between the administration of spinal anaesthesia and delivery. For inspection of summarised changes in these parameters from spinal anaesthesia to delivery, the beatwise time-series were processed as follows. First, every 76 continuous repeated measurements plots (MatLab) were inspected visually and time-series with technical errors were excluded from the analyses $(n=5)$. Second, the timeseries were smoothed by a median filter with a window length of 60 samples and truncation at edges. Finally, the measurements were aligned between participants by assigning each measurement within intervals of exactly 5 $\mathrm{s}$ and taking the mean within these intervals.
Owing to differences in recording lengths, one period of data were extracted from $3 \mathrm{~min}$ before spinal anaesthesia, until $3 \mathrm{~min}$ after. A second period was extracted from $3 \mathrm{~min}$ before delivery until delivery. The mean and SD between participants were used to present the relevant summarised changes in all parameters. The time-series at the individual level are also made available as electronic supplementary.

To compare haemodynamic values before and after spinal anaesthesia but close to delivery of the baby, we calculated the data pairs for CO, SAP and HR defined as the median of unfiltered data values within the last $30 \mathrm{~s}$ until spinal anaesthesia, and the median of values within the last $30 \mathrm{~s}$ before delivery. Owing to technical error in monitor data within the selected time window, one 
Table 1 Clinical characteristics

Median (range)/N (\%)/

mean \pm SD

\begin{tabular}{ll}
\hline Age (years) & $34.5(21-43)$ \\
\hline BMI, kg/m² & $28.5 \pm 4.3$ \\
\hline Nulliparous & $17(22 \%)$ \\
GA, weeks & $39(36-40)$ \\
\hline Preoperative $\mathrm{Hb}, \mathrm{g} / \mathrm{L}$ & $113 \pm 11$ \\
\hline Preoperative fasting, hours & $12.0 \pm 2.8$ \\
\hline Baseline & \\
\hline Systolic AP, mm Hg & $134 \pm 14.0$ \\
\hline Mean AP, mm Hg & $91 \pm 9.2$ \\
\hline Diastolic AP, mm Hg & $68 \pm 7.5$ \\
\hline HR, beats/min & $77 \pm 11.6$ \\
\hline
\end{tabular}

Clinical characteristics of 76 patients.

*Baseline values representing a 60 s mean of intra-arterial blood pressure and heart rate with the patient in left lateral position. AP, arterial pressure; BMI, body mass index; GA, gestational age; $\mathrm{HR}$, heart rate.

patient was excluded (figure 1). These data pairs were compared statistically using the paired samples t-test for normally distributed data or the Wilcoxon Signed Rank test otherwise (based on the Shapiro-Wilk test). For each parameter, CIs were calculated for the differences in mean values and for differences in medians based on the bootstrap method (BCA type, using the bootci() function in Matlab). Data processing and statistical analysis were done in Matlab2019b and SigmaPlot V.14.0.

\section{RESULTS}

Inclusion of participants is described in figure 1. Baseline measurements of HR, SAP, MAP, DAP, BMI, age, parity and gestational age for the 76 included women are presented in table 1 .

Comparing the data pairs before spinal anaesthesia with before delivery, the median difference in $\mathrm{CO}$ was $0.17 \mathrm{~L} /$ min with a $95 \%$ CI from -0.15 to $0.53 \mathrm{~L} / \mathrm{min}$. The median $\mathrm{CO}$ was $6.51 \mathrm{~L} / \mathrm{min}$ before spinal anaesthesia and $6.40 \mathrm{~L} /$ min just before delivery with IQRs of 5.56-7.54 and 5.837.56 , respectively, with no statistically significant difference between these levels $(\mathrm{p}=0.40$, Wilcoxon signed rank test; figure 2). SAP increased with a median difference of $9.0 \mathrm{~mm} \mathrm{Hg}$ (95\% CI from $2.3 \mathrm{~mm} \mathrm{Hg}$ to $13.0 \mathrm{~mm} \mathrm{Hg}$ ), increasing from $128.5 \mathrm{~mm} \mathrm{Hg}$ to $134.1 \mathrm{~mm} \mathrm{Hg}$ with IQRs of 120.1-142.7 and 124.0-146.6, respectively ( $\mathrm{p}=0.014$, Wilcoxon signed rank test). HR decreased with a mean difference of 10.8 (95\% CI from 8.2 to 13.4), decreasing from 86.0 to 75.2 with SD of 13.9 and 14.2, respectively $(\mathrm{p}<0.001$, paired t-test) The changes in $\mathrm{CO}$ for all individuals after induction of spinal anaesthesia and before delivery is presented in figure 3 . The changes in the three parameters from before spinal anaesthesia to before delivery are presented in figure 4 , and the individual
A)

Systolic AP around spinal anesthesia

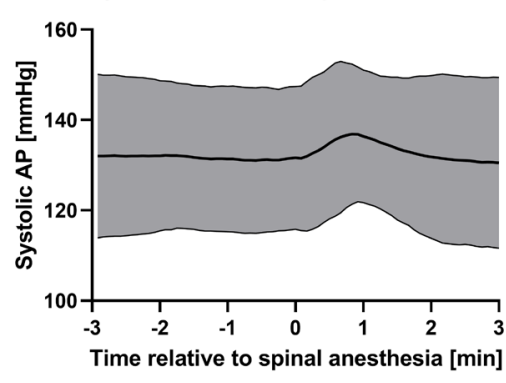

C)

$H R$ around spinal anesthesia

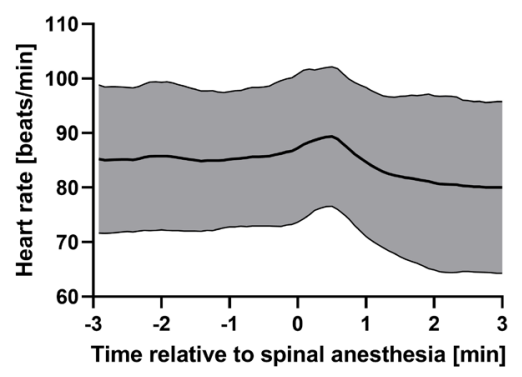

E)

$\mathrm{CO}$ around spinal anesthesia

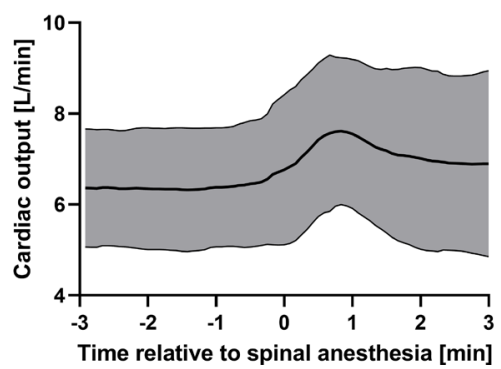

B)

Systolic AP until delivery

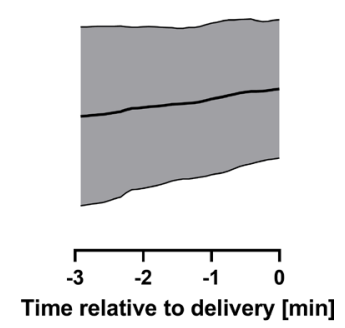

D)

HR until delivery

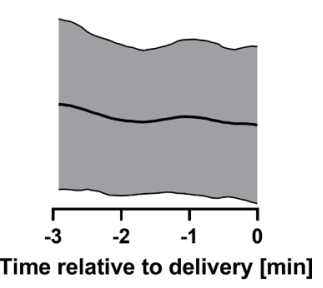

F)

CO until delivery

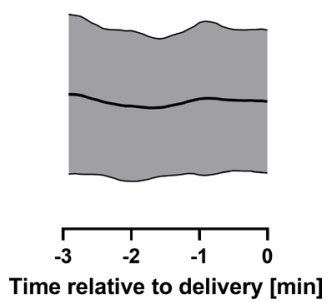

Time relative to delivery [min]

Figure 2 Systolic AP, HR and $\mathrm{CO}$ at the time of spinal anaesthesia and at delivery $(\mathrm{N}=71)$. Level of systolic AP, $\mathrm{HR}$ and $\mathrm{CO}$ around the time that spinal anaesthesia was given ( $A, C$ and $E)$, and for the last minutes until delivery (B, D and F). The thick solid line marks the mean over all participants, and the grey area represents $\pm \mathrm{SD}$. AP, arterial pressure; CO, cardiac output; HR, heart rate.

data for SAP, HR and CO are presented in figures 5-7, respectively.

After spinal anaesthesia combined with low-dose intravenous phenylephrine, we observed the following changes: (1) mean SAP increased slightly (figures 2A and 5) and remained largely unchanged until delivery (figure 2); (2) HR decreased and remained lower until delivery (figures 2C,D and 6) and (3) mean CO increased after spinal anaesthesia (figures $2 \mathrm{E}$ and 7 ) but decreased to baseline values after approximately $2 \mathrm{~min}$ and was close to baseline values at delivery (figure $2 \mathrm{~F}$ ).

\section{DISCUSSION}

Using continuous invasive monitoring during caesarean section, we found that $\mathrm{CO}$ was not significantly different 
A)

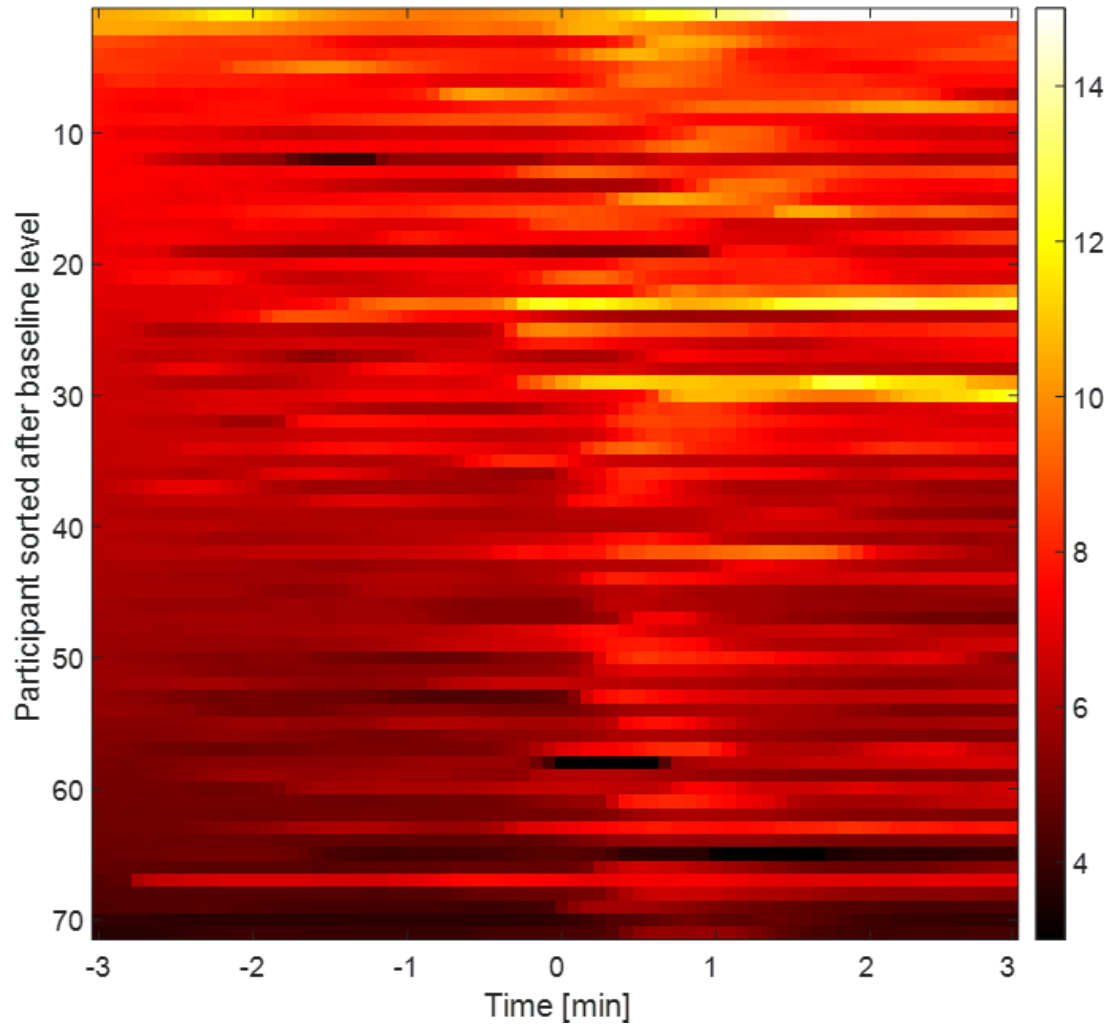

B)

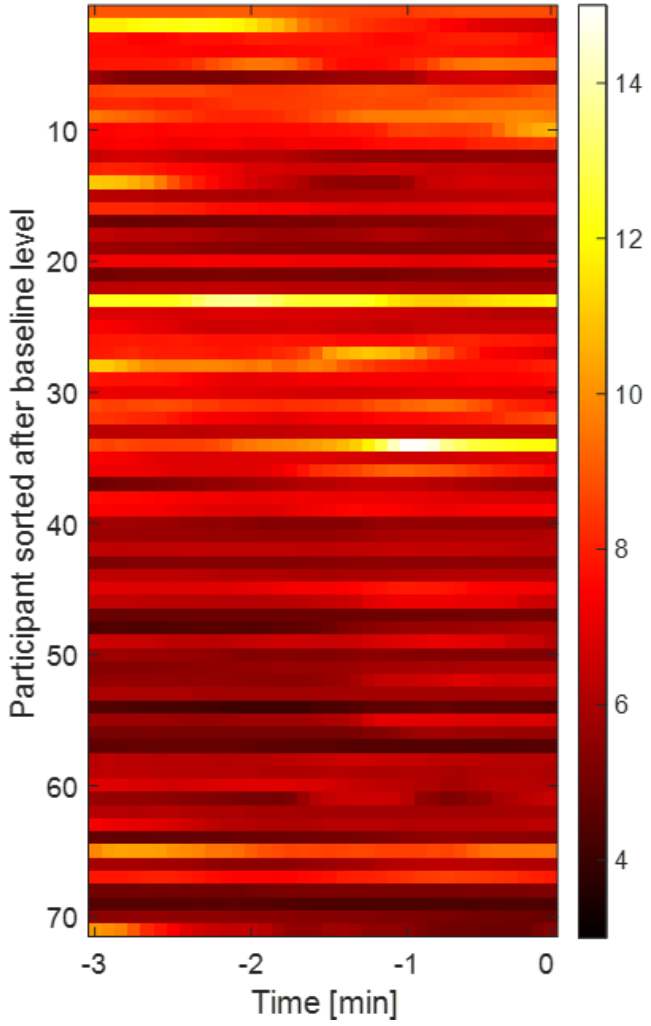

Figure 3 Alterations in $\mathrm{CO}$ before and after induction of spinal anaesthesia and until delivery in every participant (N=71). (A) Time relative to induction of spinal anaesthesia with participants on the vertical axis and $\mathrm{CO}$ levels described by colours as described in the scale to the right. Participants are ranked from highest to lowest based on CO levels at $-3 \mathrm{~min}$. (B) Time relative to delivery with participants on the vertical axis and $\mathrm{CO}$ levels described by colours as described in the scale to the right. Participants are ranked from highest to lowest based on $\mathrm{CO}$ levels at $-3 \mathrm{~min}$ in (A). Darker colour indicates lower $\mathrm{CO}$ and lighter colour indicates higher $\mathrm{CO}$. $\mathrm{CO}$, cardiac output.

at delivery compared with prior to induction of spinal anaesthesia, while SAP levels were slightly higher. The mean HR decreased and remained lower than the preanaesthetic levels until delivery.

The study has several strengths. We report measurements of vital circulatory parameters measured invasively and continuously in a relatively large cohort of women. Invasive measurement of blood pressure is the gold standard methodology and both blood pressure and HR were measured with high accuracy. The women were healthy, scheduled for planned caesarean section and the observations are generalisable. All participating women were treated according to a standardised departmental procedure, the same that was used in the studies of transfer of nutrients between mother, placenta and fetus in vivo. ${ }^{34}$ $\begin{array}{ll}\text { A) } & \text { B) } \\ \text { SAP } & \text { CO }\end{array}$

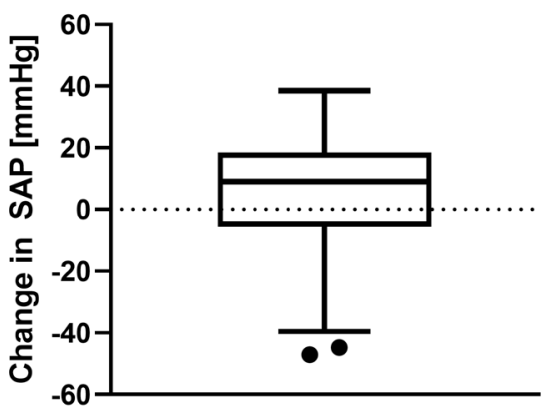

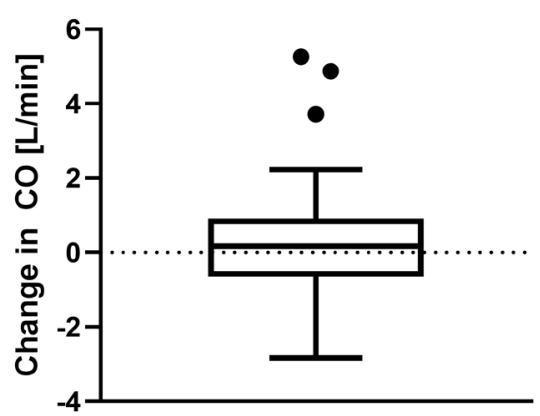

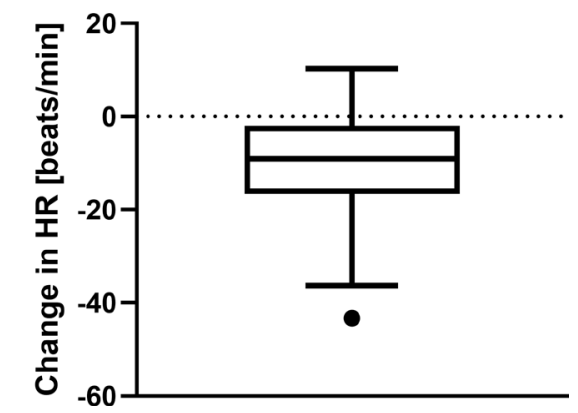

Figure 4 Changes in maternal SAP, CO and HR from before induction of spinal anaesthesia compared with before delivery. changes in median of (A) SAP, (B) CO and (C) HR from just before induction of spinal anaesthesia to just before delivery, presented in boxplots according to the Tukey method $(\mathrm{N}=70)$. $\mathrm{CO}$, cardiac output; HR, heart rate; SAP, systolic arterial pressure. 

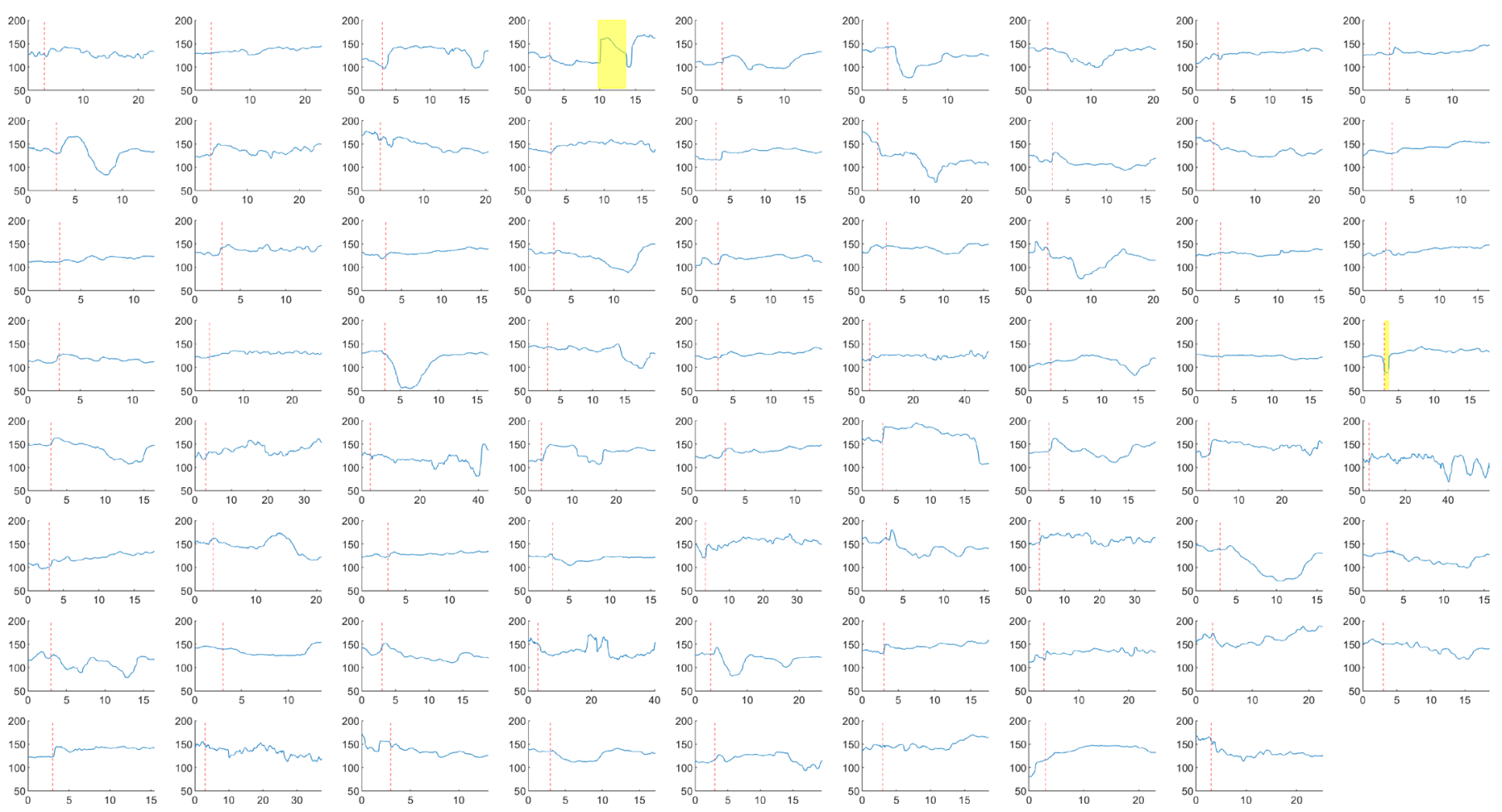

Figure 5 Individual systolic arterial blood pressure data for all 71 included participants from induction of spinal anaesthesia to delivery. Individual systolic arterial pressure data in $\mathrm{mm} \mathrm{Hg}$ for each participant from 3 min before induction of spinal anaesthesia until delivery. The vertical, red-dashed line indicates the time of spinal administration. Yellow fields indicate periods with low technical quality. These periods were not part of the calculations. All recordings are filtered using a median filter with a window of 60 beats. Time on the horizontal axis in minutes.

The study also has some limitations. CO was measured without calibration as the aim of the study was to analyse within subject trends and repeated measurements changes. The individual values measured should not be interpreted as exact, but the trending abilities of the LiDCO method are good compared with other invasive techniques. ${ }^{14}$ The spinal anaesthesia doses were equal and the vasoconstrictor protocol was consistent in all participants, but individual adjustments were necessary to keep SAP stable. The fact that the study was conducted in a setting of low-dose bupivacaine spinal anaesthesia, lateral block placement and bolus plus phenylephrine infusion in women with a median BMI of 28 at delivery may limit the generalisability of the study. The observational design did not allow comparisons of effects on $\mathrm{CO}$ between different spinal anaesthesia or vasopressor regimens. The statistical power was adequate to detect a statistically significant increase in SAP and a decrease in HR. For $\mathrm{CO}$, the median change of $0.17 \mathrm{~L} / \mathrm{min}$ was not statistically significant $(\mathrm{p}=0.40)$, and a larger sample could potentially detect a true difference. However, the 95\% CIs of the median change in CO from our sample $(-0.15 \mathrm{~L} / \mathrm{min}$ to $0.53 \mathrm{~L} / \mathrm{min})$ is narrow compared with what we consider clinically important, indicating that a larger sample size would probably not provide estimates of importance nor affect the conclusion of the study.

The primary objective was to determine whether $\mathrm{CO}$ close to delivery is comparable with preanaesthetic levels. Our measurements indicated an immediate effect of spinal anaesthesia on maternal circulatory parameters and that the low-dose phenylephrine prophylaxis protocol successfully counteracted these changes. As a result, $\mathrm{CO}$ remained stable. Based on these findings, it is reasonable to deduce that $\mathrm{CO}$ measured before induction of spinal anaesthesia predicts $\mathrm{CO}$ at delivery. This is relevant for the project in which transfer of nutrients between mother, placenta and fetus in vivo is being investigated. ${ }^{34}$

A few studies have examined effects of spinal anaesthesia on vital parameters in pregnant women. Lato $e \mathrm{al}^{15}$ evaluated 40 women by Doppler sonography before and immediately after spinal anaesthesia. They found that in $90 \%$ of the women, blood pressure decreased significantly after spinal anaesthesia and that $43 \%$ of the women experienced severe hypotension. They found a significant negative correlation between maternal blood pressure change and resistance index of the umbilical artery, and concluded that healthy fetuses seemed to compensate well when exposed to decreased uteroplacental blood flow. ${ }^{15}$ The fact that the authors observed severe hypotension in $>40 \%$ of the patients, raises the question as to whether the regimen, which was different from in the present study, was sufficient to prevent hypotension. It is not clear whether the participating women were positioned in a left lateral tilt position, the authors did not use invasive monitoring, and it is difficult to compare the results to our study. In line with the findings of Lato et al,${ }^{15}$ GuedesMartins et $a l^{16}$ found that spinal anaesthesia induced hypotension at caesarean section, but that hypotension 

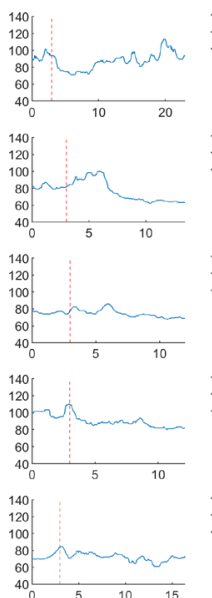

${ }^{140}+$

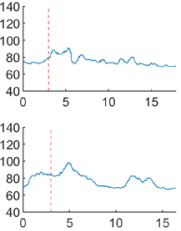

140
120
100
80

100
80
60
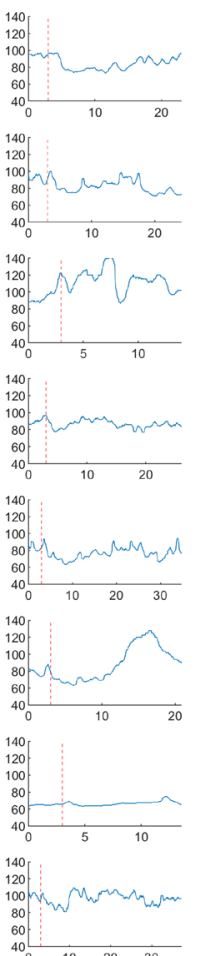
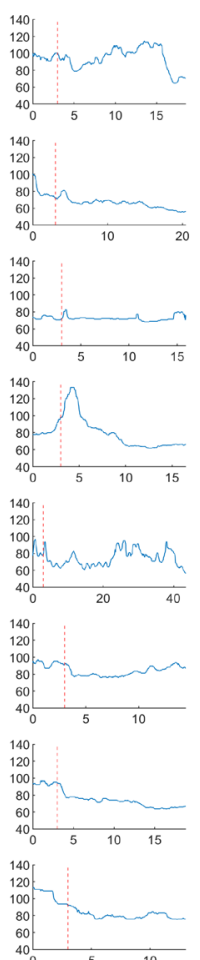
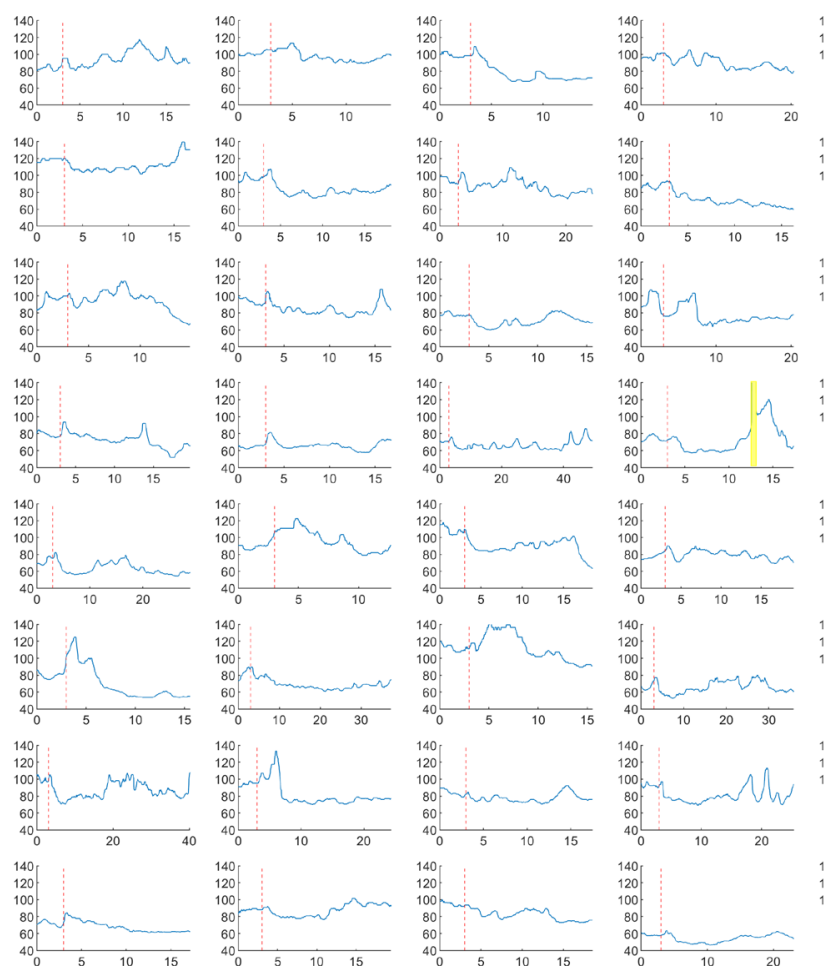
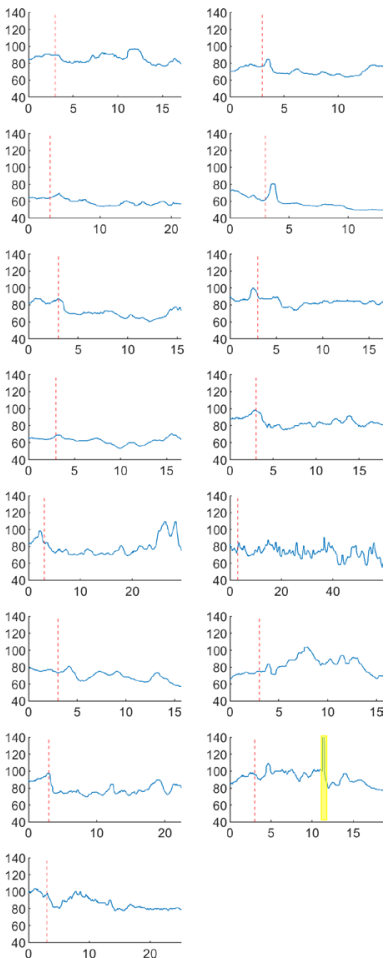

Figure 6 Individual heart rate data for all 71 included participants from induction of spinal anaesthesia to delivery. Individual heart rate data in $\mathrm{mm} \mathrm{Hg}$ for each participant from $3 \mathrm{~min}$ before induction of spinal anaesthesia until delivery. The vertical, red-dashed line indicates the time of spinal administration. Yellow fields indicate periods with low technical quality. These periods were not part of the calculations. All recordings are filtered using a median filter with a window of 60 beats. Time on the horizontal axis in minutes.
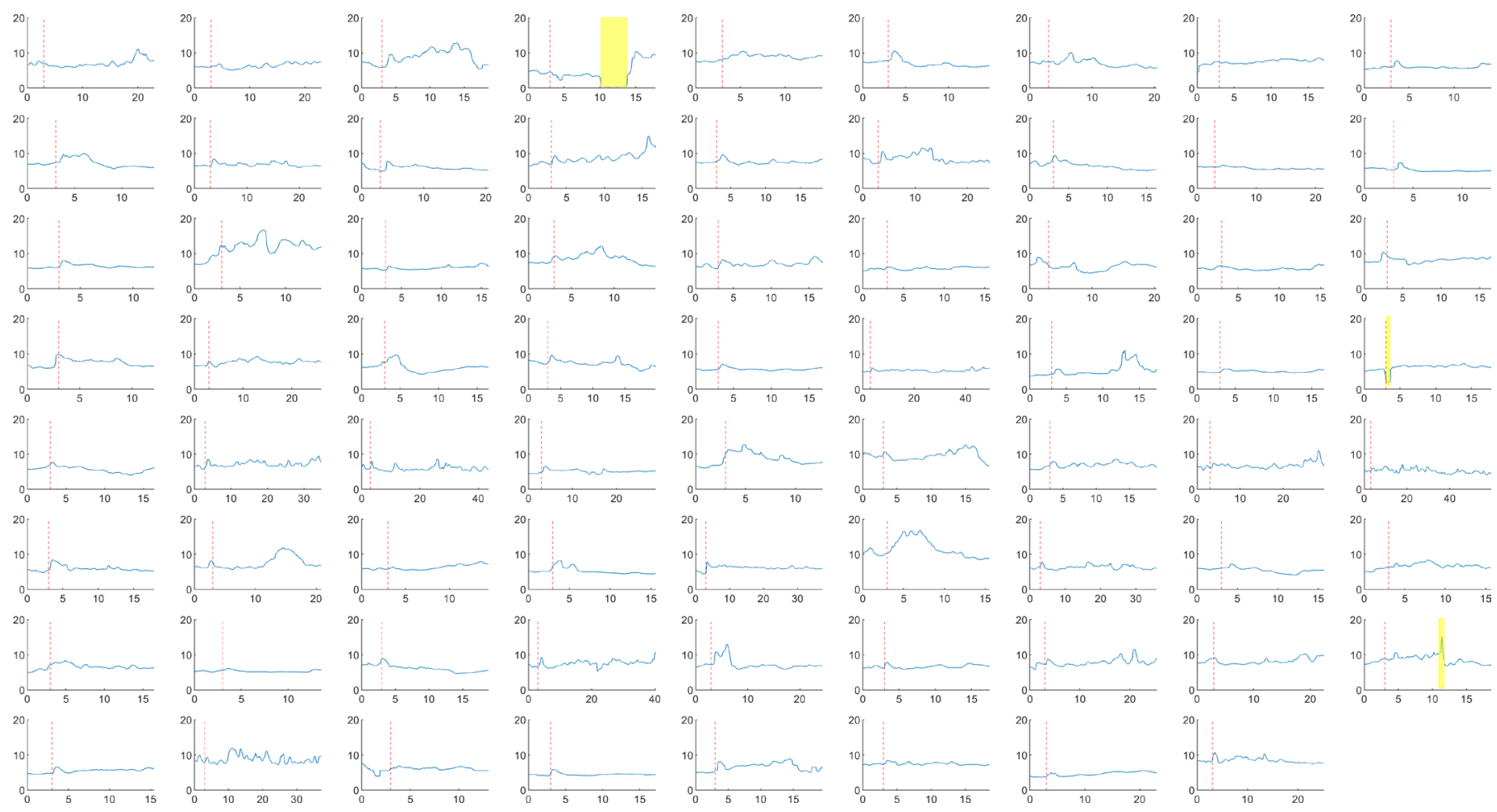

Figure 7 Individual CO data for all 71 included participants from induction of spinal anaesthesia to delivery. Individual cardiac output data in $\mathrm{mm} \mathrm{Hg}$ for each participant from 3 min before induction of spinal anaesthesia until delivery. The vertical, reddashed line indicates the time of spinal administration. Yellow fields indicate periods with low technical quality. These periods were not part of the calculations. All recordings are filtered using a median filter with a window of 60 beats. Time on the horizontal axis in minutes. 
was not associated with altered pulsatility indices in the uterine or umbilical arteries.

Guedes-Martins et $a l^{16}$ examined blood pressure and uterine and umbilical artery pulsatility indices before and after spinal anaesthesia in normotensive and hypertensive women without pre-eclampsia. They found a decrease in MAP after spinal anaesthesia in both study groups, while the fetal circulatory parameters were unchanged. Their results are in contrast to our findings of a small increase in blood pressure, but may be explained by the fact that Guedes-Martins et $a l^{16}$ did not infuse a vasoconstrictor to avoid spinal anaesthesia-induced hypotension. Their study supports that vasoconstrictor prophylaxis is necessary to avoid maternal hypotension. At the same time, their findings indicate that even though maternal circulatory parameters are affected by induction of spinal anaesthesia, there seem to be mechanisms that protect the fetus against diminished blood flow.

The efficacy of the protocol used in our institution has been documented previously. ${ }^{17} \mathrm{~A} 70 \mathrm{~kg}$ woman will receive $17 \mu \mathrm{g} / \mathrm{min}$ of phenylephrine in our hospital. Other researchers have infused up to $50 \mu \mathrm{g} / \mathrm{min},{ }^{18} 100 \mu \mathrm{g} / \mathrm{min}^{19}$ or intermittent boluses of as much as $1 \mu \mathrm{g} / \mathrm{kg} .{ }^{20}$ Clearly, these high-dose regimens will effectively prevent hypotension, but $\mathrm{HR}$ and $\mathrm{CO}$ will decrease correspondingly. ${ }^{21}$

We observed a decrease in HR and an increase in SAP. To fully understand the effects on the haemodynamics of spinal anaesthesia it is necessary to follow the individual cases beat-by-beat (figures 5-7). Even in this perspective, the moderate dose of bupivacaine in the spinal anaesthesia combined with the low-dose phenylephrine protocol seems to prevent the spinal anaesthesia-induced hypotension. This finding is in contrast to previous findings, which reported maternal hypotension after spinal anaesthesia. ${ }^{11} 1622$

The cases published with severe bradycardia due to major hypotension are few. Theoretically, bradycardia after spinal anaesthesia may be caused by neural blockade of higher thoracic spinal sympathetic nerve fibres, leading to vasodilation and reduced ability to compensate for circulatory changes with increased HR. Baroreceptors in the carotid artery are of crucial importance in regulation of HR and BP. During spinal anaesthesia-induced hypotension, this reflex may be paradoxically active. If hypotension is severe, venous return to the heart decreases and sudden bradycardia may occur, leading to a dramatic fall in blood pressure and CO. ${ }^{23}$

In our study, only $0.25 \mu \mathrm{g} / \mathrm{kg} / \mathrm{min}$ was given and the analysis indicated that this is sufficient to maintain adequate SAP with minimal risk of deleterious effects on HR and CO. In a randomised comparison of phenylephrine $100 \mu \mathrm{g} / \mathrm{min}$ and ephedrine $5 \mathrm{mg} / \mathrm{min}$, Mon et $a l^{24}$ found that $\mathrm{CO}$ decreased in the phenylephrine group and increased in the ephedrine group. ${ }^{24}$ Prophylactic vasopressors are recommended to prevent spinal anaesthesia-induced hypotension, ${ }^{9}$ and the number of cases with bradycardia is lower if phenylephrine doses are low. However, efforts to keep blood pressure stable in all parturients may lead to a decrease in $\mathrm{CO}$ in some individuals given that $\mathrm{CO}$ depends to a high degree on the actual HR ( $\mathrm{CO}=$ heart stroke volume $\times \mathrm{HR})$. Neonatal $\mathrm{pH}$ will in general be lower with ephedrine, which should therefore not replace phenylephrine in routine hypotension prophylaxis. ${ }^{25}$

Can our findings of unchanged $\mathrm{CO}$ before spinal anaesthesia compared with $\mathrm{CO}$ at delivery support that uteroplacental flow estimated before spinal anaesthesia by ultrasound Doppler is valid at delivery? It has been estimated that the uteroplacental unit receives $3.5 \%$ of maternal CO in early pregnancy and $12 \%$ near term. The resistance to flow in the uterine vessels, measured as the peak systolic to the end-diastolic flow velocity ratio, decreases from 5.3 in the non-pregnant state to a mean of 2.3 near term. ${ }^{1}$ In a recent study, Flo $e t a l^{26}$ found that the uterine fraction of maternal CO increased from $5.6 \%$ at 22 weeks of gestation to $11.7 \%$ at term. These alterations are interpreted as a development of the uteroplacental circulation to meet the metabolic demands of the growing fetus. While the systemic vascular resistance increases after the second trimester towards term, ${ }^{27}$ the resistance in the uterine vessels decreases, and hence facilitates delivery of a higher fraction of blood towards the uterus, placenta and foetus. Whether uterine blood flow is only determined by $\mathrm{CO}$ has been questioned. Classical studies in animal models have indicated that uteroplacental blood flow is kept stable within a range of perfusion pressures under maternal treatment with vasopressor. ${ }^{28}{ }^{29}$ In healthy human pregnancies, the uteroplacental vessels are transformed into low-resistance channels through which maternal blood can enter the intervillous space with low force. The uteroplacental circulation is through evolution established to maintain stable blood flow to support fetal growth and nutritional needs. ${ }^{2}$ Fetal adaptations to the acute changes in maternal haemodynamics due to spinal anaesthesia are affected by choice of vasopressor. Most studies conducted across the last decades have found phenylephrine to be superior to ephedrine. ${ }^{30}$

\section{CONCLUSION}

Blood pressure and $\mathrm{CO}$ at delivery is comparable to values before spinal anaesthesia when spinal anaesthesia is combined with prophylactic low-dose phenylephrine infusion.

\section{Twitter Leiv Arne Rosseland @r-leiv}

Contributors TMM, CT and LAR: contributed to conception and design of the work. CT: performed analyses. TMM, CT and LAR: interpreted data. TMM and LAR: drafted the work; and TMM, CT and LAR: revised the work critically for important intellectual content. All authors approved the version published and are accountable for all aspects related to the accuracy and integrity of any part for the work.

Funding The authors have not declared a specific grant for this research from any funding agency in the public, commercial or not-for-profit sectors.

Competing interests None declared.

Patient and public involvement Patients and/or the public were not involved in the design, or conduct, or reporting, or dissemination plans of this research. 
Patient consent for publication Not required.

Ethics approval The protocol was approved by the Data Inspectorate's local representative at Oslo University Hospital, the Regional Committee for Medical and Health Research Ethics of Southern Norway (Oslo, Norway; 2009/130) and the Norwegian Medicines Agency (Oslo, Norway; 09/07301-7). This approval covered both the RCT and this observational study. All data collected in this substudy before the Investigational Medicinal Product were given.

Provenance and peer review Not commissioned; externally peer reviewed.

Data availability statement Data are available in a public, open access repository. The project is completed, the dataset is anonymised and all data analysed in the present project are available open access (uploaded in Mendeley (Rosseland, Leiv Arne (2020), "Dataset C Section Hemodynamic", Mendeley Data, v2 http://dx.doi. org/10.17632/f5cmz9npjw.2)).

Open access This is an open access article distributed in accordance with the Creative Commons Attribution Non Commercial (CC BY-NC 4.0) license, which permits others to distribute, remix, adapt, build upon this work non-commercially, and license their derivative works on different terms, provided the original work is properly cited, appropriate credit is given, any changes made indicated, and the use is non-commercial. See: http://creativecommons.org/licenses/by-nc/4.0/.

ORCID iD

Trond Melbye Michelsen http://orcid.org/0000-0001-9534-6255

\section{REFERENCES}

1 Thaler I, Manor D, Itskovitz J, et al. Changes in uterine blood flow during human pregnancy. Am J Obstet Gynecol 1990;162:121-5.

2 Degner K, Magness RR, Shah DM. Establishment of the human Uteroplacental circulation: a historical perspective. Reprod Sci 2017;24:753-61.

3 Michelsen TM, Henriksen T, Reinhold D, et al. The human placental proteome secreted into the maternal and fetal circulations in normal pregnancy based on 4-vessel sampling. Faseb J 2019;33:2944-56.

4 Michelsen TM, Holme AM, Holm MB, et al. Uteroplacental glucose uptake and fetal glucose consumption: a quantitative study in human pregnancies. J Clin Endocrinol Metab 2019;104:873-82.

5 Cyna AM, Dodd J. Clinical update: obstetric anaesthesia. Lancet 2007;370:640-2.

6 Rollins M, Lucero J. Overview of anesthetic considerations for cesarean delivery. Br Med Bull 2012;101:105-25.

7 Ralston DH, Shnider SM. The fetal and neonatal effects of regional anesthesia in obstetrics. Anesthesiology 1978;48:34-64.

8 Gibbs MW, Van Dyk D, Dyer RA. Managing spinal hypotension during caesarean section: an update. S Afr Med J 2018;108:460-3.

9 Fitzgerald JP, Fedoruk KA, Jadin SM, et al. Prevention of hypotension after spinal anaesthesia for caesarean section: a systematic review and network meta-analysis of randomised controlled trials. Anaesthesia 2020;75:109-21.

10 Robson SC, Boys RJ, Rodeck C, et al. Maternal and fetal haemodynamic effects of spinal and extradural anaesthesia for elective caesarean section. Br J Anaesth 1992;68:54-9.

11 Cyna AM, Andrew M, Emmett RS, et al. Techniques for preventing hypotension during spinal anaesthesia for caesarean section. Cochrane Database Syst Rev 2006;4:CD002251.

12 Langesaeter E, Rosseland LA, Stubhaug A. Continuous invasive blood pressure and cardiac output monitoring during cesarean delivery: a randomized, double-blind comparison of low-dose versus high-dose spinal anesthesia with intravenous phenylephrine or placebo infusion. Anesthesiology 2008;109:856-63.
13 Rosseland LA, Hauge TH, Grindheim G, et al. Changes in blood pressure and cardiac output during cesarean delivery: the effects of oxytocin and carbetocin compared with placebo. Anesthesiology 2013;119:541-51.

14 Hadian M, Kim HK, Severyn DA, et al. Cross-comparison of cardiac output trending accuracy of LiDCO, PiCCO, FloTrac and pulmonary artery catheters. Crit Care 2010;14:R212.

15 Lato K, Bekes I, Widschwendter P, et al. Hypotension due to spinal anesthesia influences fetal circulation in primary caesarean sections. Arch Gynecol Obstet 2018;297:667-74.

16 Guedes-Martins L, Graça H, Saraiva JP, et al. The effects of spinal anaesthesia for elective caesarean section on uterine and umbilical arterial Pulsatility indexes in normotensive and chronic hypertensive pregnant women: a prospective, longitudinal study. BMC Pregnancy Childbirth 2014;14:291.

17 Kuhn JC, Hauge TH, Rosseland LA, et al. Hemodynamics of phenylephrine infusion versus lower extremity compression during spinal anesthesia for cesarean delivery: a randomized, double-blind, placebo-controlled study. Anesth Analg 2016;122:1120-9.

18 Orbach-Zinger S, Razinsky E, Bizman I, et al. Perioperative noninvasive cardiac output monitoring in parturients with singleton and twin pregnancies undergoing cesarean section under spinal anesthesia with prophylactic phenylephrine drip: a prospective observational cohort study. J Matern Fetal Neonatal Med 2019;32:3980-5.

19 Ngan Kee WD, Khaw KS, Ng FF. Comparison of phenylephrine infusion regimens for maintaining maternal blood pressure during spinal anaesthesia for caesarean section. Br J Anaesth 2004;92:469-74.

20 Lee H-M, Kim S-H, Hwang B-Y, et al. The effects of prophylactic bolus phenylephrine on hypotension during low-dose spinal anesthesia for cesarean section. Int J Obstet Anesth 2016;25:17-22.

21 Stewart A, Fernando R, McDonald S, et al. The dose-dependent effects of phenylephrine for elective cesarean delivery under spinal anesthesia. Anesth Analg 2010;111:1230-7.

22 Mercier FJ, Augè M, Hoffmann C, et al. Maternal hypotension during spinal anesthesia for caesarean delivery. Minerva Anestesiol 2013;79:62-73.

23 Kinsella SM, Tuckey JP. Perioperative bradycardia and asystole: relationship to vasovagal syncope and the Bezold-Jarisch reflex. $\mathrm{Br} \mathrm{J}$ Anaesth 2001;86:859-68.

24 Mon W, Stewart A, Fernando R, et al. Cardiac output changes with phenylephrine and ephedrine infusions during spinal anesthesia for cesarean section: a randomized, double-blind trial. J Clin Anesth 2017;37:43-8.

25 Ngan Kee WD, Lee A. Multivariate analysis of factors associated with umbilical arterial $\mathrm{pH}$ and standard base excess after caesarean section under spinal anaesthesia. Anaesthesia 2003;58:125-30.

26 Flo K, Wilsgaard T, Vårtun A, et al. A longitudinal study of the relationship between maternal cardiac output measured by impedance cardiography and uterine artery blood flow in the second half of pregnancy. BJOG 2010;117:837-44.

27 Sanghavi M, Rutherford JD. Cardiovascular physiology of pregnancy. Circulation 2014;130:1003-8.

28 Buss DD, Bisgard GE, Rawlings CA, et al. Uteroplacental blood flow during alkalosis in the sheep. Am J Physiol 1975;228:1497-500.

29 Venuto RC, Cox JW, Stein JH, et al. The effect of changes in perfusion pressure on uteroplacental blood flow in the pregnant rabbit. J Clin Invest 1976;57:938-44.

30 Habib AS. A review of the impact of phenylephrine administration on maternal hemodynamics and maternal and neonatal outcomes in women undergoing cesarean delivery under spinal anesthesia. Anesth Analg 2012;114:377-90. 